\title{
Asynchronous Detected Disappearance of the Pro-nuclei by Time-Lapse X Embryonic Development
}

\author{
Guilherme Rios Franco Rosário ${ }^{1}$, Antônio Carlos Costa Franco ${ }^{1}$, Adriana Valério da Silva ${ }^{1}$ \\ ${ }^{1}$ Embryolife Instituto de Medicina Reprodutiva, São José dos Campos/SP.
}

\begin{abstract}
Objective: To investigate whether pro-nuclei disappearance synchronicity would have something to do with blastomere cleavage time up to D3 (timeline) and if it would be associated with the appearance of embryo abnormalities. Methods: The sample of the present study was designed with 100 embryos $(n=100)$. With the aid of an embryonic analysis equipment: Time-Lapse, we studied embryonic morphokinetics from the zygote stage to D3.

Results: When there was no pronuclei synchronous disappearance, $30.8 \%$ underwent normal cleavage, whereas when there was pronuclei synchronous disappearance, $44.8 \%$ had normal cleavage. When there was no pronuclei synchronous disappearance, $46.2 \%$ had some type of abnormality, whereas when there was pronuclei synchronous disappearance, $56.3 \%$ had some kind of abnormality.

Conclusion: Although the statistical results were not significant, we believe that with a larger sample size we could state with higher reliability, that the pro-nuclei synchronous disappearance analysis may, in the near future, indicate higher embryo quality.
\end{abstract}

Keywords: Embryo, Time-Lapse, Abnormalities, Cleavage, In vitro fertilization.

\section{INTRODUCTION}

Embryo development analysis by Time-Lapse is an important method to complement embryo selection, which combined with classical morphological classification may further improve the precise choice of embryos with a higher implantation potential. Several authors describe specific morphokinetic parameters in embryos with greater likelihood of implantation (Lemmen et al., 2008; Wong et al., 2010; Meseguer et al., 2011; Cruz et al., 2012; Dal Canto et al., 2012; Hashimoto et al., 2012; Basile et al., 2015). With this technique, the development of quality blastocysts may be predicted within the first 48 hours of culture (Kirkegaard et al., 2013).

It is certain that the continuous monitoring of embryos opens opportunities, making it possible to fully assess the events significant for best embryo selection. Happenings associated with low implantation rates, such as multinucleation (Pickering et al., 1995), asymmetric blastomeres (Hardarson et al., 2001), direct cleavage to 3 cells (1C-3C) (Rubio et al., 2012 ) and fragmentation (Alikani et al., 1999) occur dynamically and continuously during embryonic development (Shawn et al., 2012). In addition to these abnormalities, we still have pronuclei kinetics, where we also have successive variations that can be seen through Time-Lapse morphokinetic analysis.

Prior to having this tool, those pronuclei distant from each other were associated with delayed cleavages and poor quality embryos (Nagy et al., 2002). We now know that the pro-nuclei can migrate through the cytoplasm, and thus those previously seen distant, will be near in another checking time. This proves that fertilization checking at a single moment may be insufficient in comparison with the Time-Lapse analysis (Azarello et al., 2012).
Upon watching the videos, we noticed that in some cases the two pronuclei upon syngamy, disappeared asynchronously, i.e., one at a time. This led us to question whether this asynchronous pro-nuclei disappearance, would have something to do with the time of blastomere cleavage to D3 (timeline), and whether there is any association with embryo abnormalities.

\section{MATERIALS AND METHODS}

The sampling of this study was designed with $100 \mathrm{em}-$ bryos $(n=100)$, all obtained by Intracytoplasmic Sperm Injection (ICSI).

The embryos were evaluated by a detailed Time-Lapse analysis (10/10min), assessing the exact time of the events and their development after ICSI.

\section{ICSI}

ICSI was performed on culture medium containing HEPES. Carried out under 250x magnification, with a Nikon ${ }^{\circledR}$ Eclipse TE 2000-S microscope with the relevant temperature variations of the central vinyl surface of the micro-handler table being controlled with a GMH 3230 Greisinger $\AA$ (made in Germany) surface thermometer, with validated calibration. After the ICSI, we were careful to "rinse" the embryos, using the same culture medium to which they were subjected in their development. The rinsing was carried out in at least 3 drops $(\sim 50 \mu \mathrm{l})$ in continuous pre-equilibrated culture medium. Soon after, the oocytes were placed in micro-wells from the special TimeLapse plate, and taken to the incubator.

\section{Incubation}

We used the same culture medium for all embryos in the present study. The $\mathrm{CO}^{2}$ level was as indicated by the manufacturer of the medium, while maintaining $\mathrm{O}^{2}$ level $\sim 20 \%$.

We used the 9- or 16- micro-well plates, prepared and pre-equilibrated in the incubator. After pre-equilibration, all micro-bubbles were carefully removed. Using the Time-Lapse technique, the incubator door was not opened during the entire embryonic development period.

\section{Image Capture System}

The images were captured by a microscope camera placed inside a "big box" type incubator. The photos were taken every 10 minutes in order to build the "timeline". The system uses a homogeneous green LED light as the light source.

Recalling that the embryos are spherical, we took photos in different planes (slices) at every 30 minutes on the first day of development in order to properly locate the pronuclei, although it was not difficult to see them even without the help of slices. On the second and third days, the photos were taken at every two hours, for the purpose of identifying multi-nucleation, the precise number of blastomeres, and finally, the presence and levels of embryonic fragmentation. 
Time-Lapse evaluation of morphokinetic parameters

We used the computer to do a retrospective analysis of the images, using a specific software that records the events that occurred after ICSI, identifying the precise timing of the pro-nuclei dissipation, cell divisions and arising abnormalities.

The exact timing of the pro-nuclei disappearance, being an event that has a relatively short duration, could be identified thanks to the Time-Lapse system. The software enabled us to identify zygotes that would present pro-nuclei disappearance simultaneously or not.

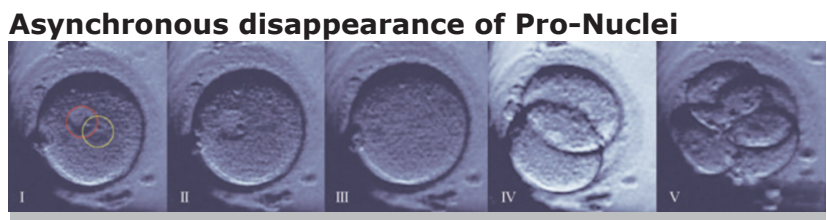

Figure 1.* After asynchronous disappearance continues as normal embryonic development, even in this case without fragmentation levels, or any other visible anomaly.

1. Two pro-nuclei highlighted in the cell cytoplasm, and their nucleoli also displayed.

2. Pronucleus it was marked yellow in the previous figure (1) disappears before the other. In this case the difference lasted for $\sim 20$ minutes.

3. Both pronuclei disappeared.

4. Cleavage occurred normally for two cells.

5. Cleavage occurred normally to four cells; the blastomeres are symmetrical, there is only one core per blastomere without fragmentation signal.

Below, we analyze an ideal cleavage curve plotted with the aid of an analysis software. To build this curve we used the first cleavage time as a parameter. Subsequently, the time of cleavage for 3 cells, 4 cells and finally 5 cells (Meseguer et al., 2011).

\section{Development Curve Graph}

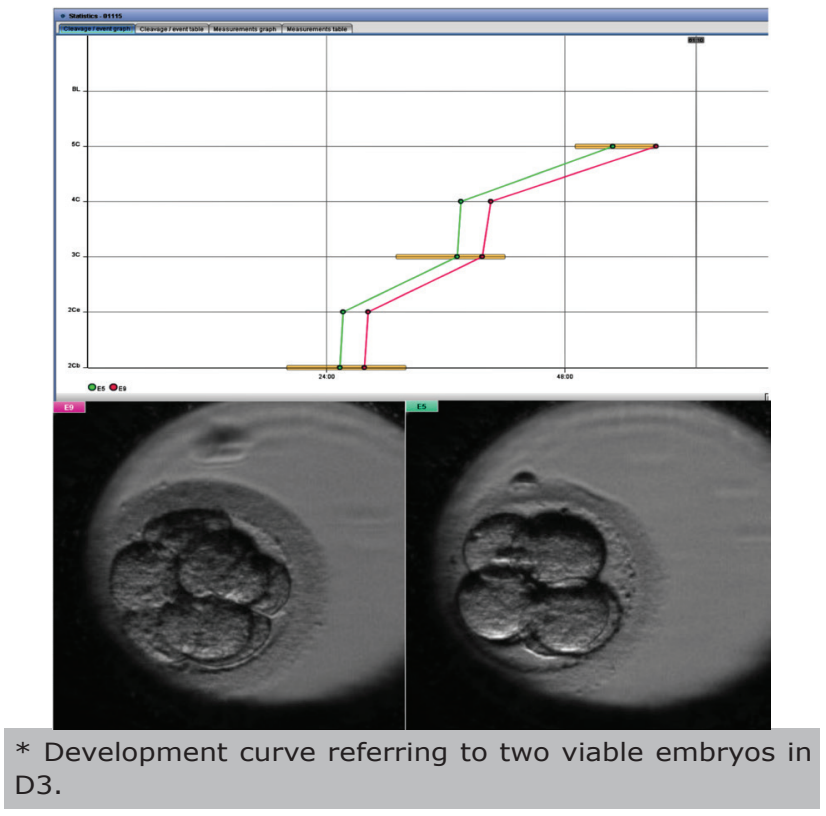

The emergence of major abnormalities such as Fragmentation, Blastomere Asymmetry, Multinucleate Blastomeres, direct Cleavage to 3 cells ( $1 \mathrm{C}-3 \mathrm{C})$, was recorded with the aid of analysis software.

\section{Statistical Analysis}

To check the effect of pro-nuclei synchronous disappearance on the timeline occurrence of abnormalities, we used the chi-square test, while for checking the effect of pro-nuclei synchronous disappearance over the types of abnormalities, we used Fisher's exact test.

As the number of abnormalities in individuals ranged from zero to three, we used the Cochran-Armitage test for trends to check whether in the group with synchronous pro-nuclei disappearance there was a tendency to have more or less abnormalities per person, compared to the group that did not have pro-nuclei synchronous disappearance.

The odds ratios and their respective confidence intervals were calculated using the median-unbiased estimation method, and should there be a zero we adjusted for small samples. 3.2.0.

The software used in the analysis was the $R$ version

\section{RESULTS}

On the Table 1, it is possible to see the pro-nuclei synchronous disappearance effect on the occurrence of embryos developing inside the timeline, on the occurrence of abnormalities, on the number of abnormalities in the same embryo and on the occurrence of different types of abnormalities. Thus, it can be noted that:

- When there was no pro-nuclei synchronous disappearance, $30.8 \%$ had normal cleavage, whereas when there was pro-nuclei synchronous disappearance, $44.8 \%$ had normal cleavage; however, this difference was not significant ( $P$-value $=0.340)$. Assessing the odds ratio, one can still state that the likelihood of regular cleavage occurring upon pro-nuclei synchronous disappearance was $1.78[0.53 ; 7.25]$ times the likelihood of when there is no synchronous disappearance.

- When there was no pro-nuclei synchronous disappearance, $46.2 \%$ had some type of abnormality, whereas when there was pro-nuclei synchronous disappearance, $56.3 \%$ had some type of abnormality, but this difference was not significant (value $P=0.492$ ). Assessing the odds ratio, one can also state that the likelihood of some kind of abnormality upon pro-nuclei synchronous disappearance was 1.49 [0.45; 5.11] times the likelihood of when there was no synchronous disappearance.

- Considering the number of abnormalities per individual and evaluating the Cochran-Armitage test for trends, there was insufficient evidence $(P$-value $=0.237)$ to state that when there is no pro-nuclei synchronous disappearance, we see a tendency towards more abnormalities per embryo.

Graph 1 - Bar chart for the occurrence of Timeline and abnormalities among pro-nuclei synchronous disappearance.
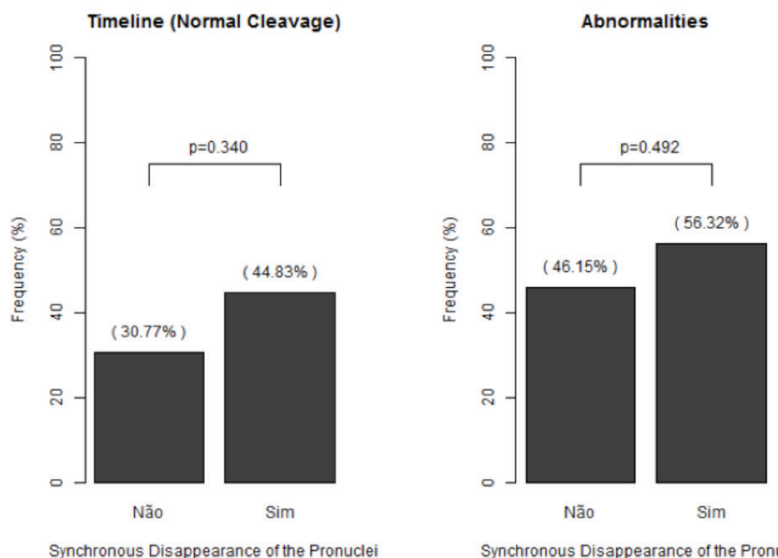

Não

Sim

Synchronous Disappearance of the Pronuclei 
Table 1. Disappearance of Synchronous Effect of Pro-cores on Timeline and Abnormalities.

\begin{tabular}{|c|c|c|c|c|c|c|c|c|c|}
\hline & Variables & & & $\begin{array}{l}\text { hronous } \\
\text { Pro- }\end{array}$ & $\begin{array}{l}\text { appe } \\
\text { uclei }\end{array}$ & rance of & $P$ & O.R. & I.C. $-95 \%$ \\
\hline & & & & $(n=13)$ & Yes & $n=87)$ & & & \\
\hline Timeline & No $(n=57)$ & & 9 & $69.2 \%$ & 48 & $55.2 \%$ & $0.340^{1}$ & 1 & - \\
\hline Normal Cleavage & Yes $(n=43)$ & & 4 & $30.8 \%$ & 39 & $44.8 \%$ & $0.340^{\circ}$ & 1.78 & {$[0.53 ; 7.25]$} \\
\hline Ahnormalities & No $(n=45)$ & & 7 & $53.8 \%$ & 38 & $43.7 \%$ & $0.492^{1}$ & 1 & - \\
\hline Futiotinaticies & Yes $(n=55)$ & & 6 & $46.2 \%$ & 49 & $56.3 \%$ & 0.472 & 1.49 & {$[0.45 ; 5.11]$} \\
\hline & None $(n=45)$ & & 7 & $53.8 \%$ & 38 & $43.7 \%$ & & 1 & - \\
\hline $\begin{array}{l}\text { Abnormalities } \\
\text { Amount }\end{array}$ & One $(n=43)$ & & 6 & $46.2 \%$ & 37 & $42.5 \%$ & $0.237^{2}$ & 1.13 & {$[0.34 ; 3.91]$} \\
\hline & Two or more ( $\mathrm{n}$ & $=12)$ & 0 & $0.0 \%$ & 12 & $13.8 \%$ & & 2.15 & {$[0.26 ; 91.5]$} \\
\hline & & No $(n=57)$ & 10 & $76.9 \%$ & 47 & $54.0 \%$ & & 1 & - \\
\hline & Fragmentation & Yes $(n=43)$ & 3 & $23.1 \%$ & 40 & $46.0 \%$ & $0.143^{\circ}$ & 2.72 & {$[0.75 ; 13.4]$} \\
\hline & Uneven & No $(n=88)$ & 12 & $92.3 \%$ & 76 & $87.4 \%$ & $1000^{3}$ & 1 & - \\
\hline & Blastomeres & Yes $(n=12)$ & 1 & $7.7 \%$ & 11 & $12.6 \%$ & $1.000^{\circ}$ & 1.55 & {$[0.26 ; 40.7]$} \\
\hline Abnormalities & & No $(n=95)$ & 13 & $100.0 \%$ & 82 & $94.3 \%$ & $1000^{3}$ & 1 & - \\
\hline Types & vacuoles & Yes $(n=5)$ & 0 & $0.0 \%$ & 5 & $5.7 \%$ & $1.000 \mathrm{o}$ & 0.78 & {$[0.09 ; 34.5]$} \\
\hline & & No $(n=96)$ & 11 & $84.6 \%$ & 85 & $97.7 \%$ & & 1 & - \\
\hline & $1 C=3 C$ & Yes $(n=4)$ & 2 & $15.4 \%$ & 2 & $2.3 \%$ & $0.081^{3}$ & 0.13 & {$[0.01 ; 1.39]$} \\
\hline & Multinucleated & No $(n=96)$ & 13 & $100.0 \%$ & 83 & $95.4 \%$ & $000^{3}$ & 1 & - \\
\hline & Blastomeres & Yes $(n=4)$ & 0 & $0.0 \%$ & 4 & $4.6 \%$ & $1.000^{\circ}$ & 0.62 & {$[0.07 ; 28.5]$} \\
\hline
\end{tabular}

${ }^{1}$ Chi Square; ${ }^{2}$ Cochran-Armitage; ${ }^{3}$ Exact Fisher. O.R. = Reason Chance; I.C.-95 = Interval 95\% confidence

Table 2. Abnormality of occurrence of the effect on the Timeline.

\section{Abnormalities}

Variables

\begin{tabular}{|c|c|c|c|c|c|c|c|c|}
\hline \multirow[b]{2}{*}{ Timeline } & \multirow[b]{2}{*}{ No $(n=57)$} & \multicolumn{2}{|c|}{ No $(n=45)$} & \multicolumn{2}{|c|}{ Yes $(n=55)$} & & & \\
\hline & & 19 & $42.2 \%$ & 38 & $69.1 \%$ & \multirow{2}{*}{$0.007^{1}$} & 1 & - \\
\hline (Normal Cleavages) & Yes $(n=43)$ & 26 & $57.8 \%$ & 17 & $30.9 \%$ & & 0.33 & {$[0.14 ; 0.75]$} \\
\hline
\end{tabular}

${ }^{1}$ Chi Square; O.R.= Reason Chance; I.C.-95 = Interval 95\% confidence

- When there was no pro-nuclei synchronous disappearance, $23.1 \%$ had fragmentation, whereas when there was pro-nuclei synchronous disappearance, $46.0 \%$ had fragmentation, but this difference was not significant ( $P$-value $=0.143$ ). Assessing the odds ratio, one can also state that the likelihood of fragmentation occurrence upon pro-nuclei synchronous disappearance was $2.72[0.75 ; 13.4]$ times the likelihood of when there was no synchronous disappearance.

Graph 2 - Bar chart for number of abnormalities upon pro-nuclei synchronous disappearance.

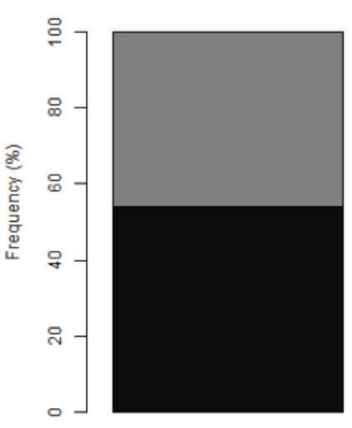

Não

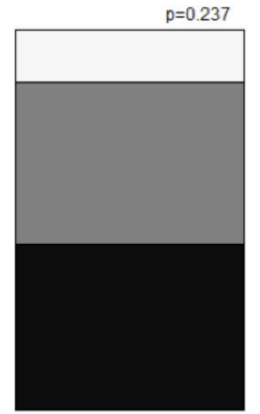

Sim
Synchronous Disappearance of the Pronuclei
- When there was no pro-nuclei synchronous disappearance, $15.4 \%$ had $1 \mathrm{C}-3 \mathrm{C}$, whereas when there was pro-nuclei synchronous disappearance, $2.3 \%$ had $1 \mathrm{C}-3 \mathrm{C}$, but this difference was marginally significant $(P$-value $=$ 0.081). Assessing the odds ratio, one can still state that the likelihood of $1 \mathrm{C}-3 \mathrm{C}$ occurrence upon pro-nuclei synchronous disappearance was $0.13[0.01 ; 1.39]$ times the likelihood of when there is no synchronous disappearance.

Graph 3 - Bar chart for the types of abnormalities with the synchronous disappearance of pro-nuclei.

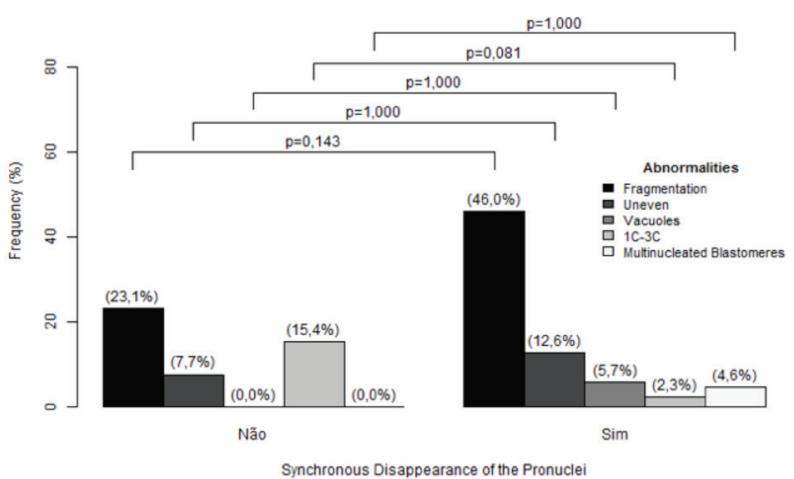


- Pro-nuclei synchronous disappearance also does not have significant influence on the occurrence of blastomere asymmetry abnormalities $(P$-value $=1.00)$, vacuoles $(P$-value $=1.00)$ and multinucleated Blastomeres $(P$-value $=1,00)$.

In the graphs 1,2 and 3 , one can revisit the information already submitted on Table 1.

On the Table 2, one can see that when there was no abnormality, $57.8 \%$ had normal cleavage, whereas when some kind of abnormality occurred, $30.9 \%$ had normal cleavage, and this difference was significant ( $P$-value = 0.007). Assessing the odds ratio, one can also state that the likelihood of normal cleavage occurrence upon the abnormality was $0.33[0.14 ; 0.75]$ times the likelihood of when there was no abnormality.

\section{DISCUSSION}

In the present study, we sought to find a connection between pro-nuclei disappearance synchrony, with subsequent embryo development. The variables investigated were Timeline and Abnormalities.

The embryos with asynchronous pro-nuclei disappearance accounted for the major portion outside the ideal cleavage curve until D3 ( 45\%). This information may be important to complement the tiebreaker criterion in the choice of embryo to be transferred, taking into account that even after this event, the embryo may still have perfect morphology. In addition, the fact that pro-nuclei disappear out of sync, may have something to do with some gamete fusion irregularity; however, this has not been scientifically proven.

We found a higher incidence of abnormalities among the embryos that had synchronous pro-nuclei disappearance. This could be due to the small sample $(n=13)$ of embryos with asynchronous disappearance. Furthermore, we did not consider seminal parameters that can increase the incidence of abnormalities, such as collection by epididymis puncture, teratospermia, DNA fragmentation and even patient age (Oliveira et al., 2014).

There was a marginally significant difference in cases where the embryos cleaved directly into 3 cells (1C-3C) with this type of cellular event being the most prevalent in the group that had asynchronous pro-nuclei disappearance $(15.4 \%)$, while in embryos with synchronous pro-nuclei disappearance, the incidence was $2.3 \%$. We know that the embryos that cleave directly $(1 \mathrm{C}-3 \mathrm{C})$ must be rejected upon embryo transfer, in order to increase the implantation rate (Rubio et al., 2012). This information reinforces the importance of observing pro-nuclei disappearance synchronicity, aiming to avoid low implantation potential embryos.

In analogy to embryonic cleavage abnormalities and timeline, we noticed that most of the embryos that fall within the ideal cleavage curve, have good morphology, corroborating literature information (Meseguer et al., 2011).

It should be noted that the sample size for the asynchronous disappearance was small $(n=13)$, which reduces the power of the tests and therefore makes it difficult to find significant results. However, with what was found in this study, we can create future prospects with a larger sample, additional data and more papers discussing embryonic morphokinetic analysis.

\section{CONCLUSIONS}

There was no significant effect of pro-nuclei synchronous disappearance of embryos on the timeline, on the occurrence of abnormalities, on the number of abnormalities in the same individual and on the occurrence of different types of abnormalities.

Despite the statistical results, we believe that with a larger sample, we can state with greater reliability that the pro-nuclei disappearance synchronicity analysis may, in the near future, indicate higher embryo quality.

\section{CONFLICT OF INTERESTS}

No conflict of interest have been declared.

\section{Corresponding author:}

Guilherme Rios Franco Rosário

Embryolife Instituto de Medicina Reprodutiva

São José dos Campos/SP

E-mail: guilherme@embryolife.com

\section{REFERENCES}

Alikani N, Jacques Cohen, Giles Tomkin, G John Garrisi, Caryn Mack, Richard T Scott. Human embryo fragmentation in vitro and its implications for pregnancy and implantation. Fertil Steril. 1999;71:836-42.

Azarello A, Hoest T, Mikkelsen AL. The impact of pronuclei morphology and dynamicity on live birth outcome after time-lapse culture. Hum. Reprod. 2012;27:2649-57.

Basile N, Vime P, Florensa M, Aparicio Ruiz B, García Velasco JA, Remohí J, Meseguer M. The use of morphokinetics as a predictor of implantation: a multicentric study to define and validate an algorithm for embryo selection. Hum. Reprod. $2015 ; 30: 276-83$

Cruz M, Garrido N, Herrero J, Perez-Cano I, Munoz M, Meseguer M. Timing of cell division in human cleavage-stage embryos is linked with blastocyst formation and quality. Reprod Biomed Online. 2012;25:371-81.

Dal Canto M, Coticchio G, Mignini Renzini M, De Ponti E, Novara PV, Brambillasca F, Comi R, Fadini R. Cleavage kinetics analysis of human embryos predicts development to blastocyst and implantation. Reprod Biomed Online. $2012 ; 25: 474-80$.

Hardarson T, Hanson C, Sjogren A, Lundin K. Human embryos with unevenly sized blastomeres have lower pregnancy and implantation rates: indications for aneuploidy and multinucleation. Hum Reprod. 2001;16:313-18.

Hashimoto S, Kato N, Saeki K, Morimoto Y. Selection of high-potential embryos by culture in poly(dimethylsiloxane) microwells and time-lapse imaging. Fertil Steril. 2012;97:332-37.

Kirkegaard K, Kesmodel US, Hindkjaer JJ, Ingerslev $\mathrm{HJ}$. Time-lapse parameters as predictors of blastocyst development and pregnancy outcome in embryos from good prognosis patients: a prospective cohort study. Hum Reprod. $2013 ; 28: 2643-51$.

Lemmen JG, Agerholm I, Ziebe S. Kinetic markers of human embryo quality using time-lapse recordings of IVF/ICSI-fertilized oocytes. Reprod Biomed Online. 2008;17:385-91.

Meseguer M, Herrero J, Tejera A, Hilligsoe KM, Ramsing NB, Remohi J. The use of morphokinetics as a predictor of embryo implantation. Hum Reprod. 2011;26:2658-71.

Nagy ZP, Dozortsev D, Diamond M, Rienzi L, Ubaldi F, Abdelmassih R and Greco E. Pronuclear morphology evaluation with subsequent evaluation of embryo morphology significantly increases implantation rates. Fertil Steril. 2003; 80:67-74.

Oliveira JBA, Petersen CG, Mauri AL, Vagnini LD, Baruffi 
R, Franco Jr. JG. The effects of age on sperm quality: an evaluation of 1,500 sêmen samples. JBRA Assist Reprod. $2014 ; 18: 34-41$

Pickering SJ, Taylor A, Johnson MH, Braude PR. An analysis of multinucleated blastomere formation in human embryos. Hum Reprod. 1995;10:1912-22.

Rubio I, Kuhlmann R, Agerholm I, Kirk J, Herrero J, Escriba MJ, Bellver J, Meseguer M. Limited implantation success of direct-cleaved human zygotes: a time-lapse study. Fertil Steril.2012;98:1458-63. Shawn C, Loewke K, Jinnuo $H$, Moussavi F, Colls P, Munne S, Behr B, Pera R. Dynamic blastomere behaviour reflects human embryo ploidy by the four-cell stage. Nature Communications. 2012;3:1251.

Wong CC, Loewke KE, Bossert NL, Behr B, De Jonge CJ, Baer TM, Reijo Pera RA. Non-invasive imaging of human embryos before embryonic genome activation predicts development to the blastocyst stage. Nat Biotechnol. 2010;28: 1115-21. 